\title{
Wearing High Heel and Planter Fasciitis: MRI Evaluation
}

\author{
Sameh Ahmad Khodair ${ }^{1,}$, , Rasha Loutfy Younes ${ }^{2}$ \\ Radiology Department, Faculty of Medicine, Tanta University, Tanta, Egypt \\ Email address: \\ samehkhodair@gmail.com (S. A. Khodair), rasha.younis@med.tanta.edu.eg (R. L. Younes) \\ ${ }^{*}$ Corresponding author
}

\section{To cite this article:}

Sameh Ahmad Khodair, Rasha Loutfy Younes. Wearing High Heel and Planter Fasciitis: MRI Evaluation. International Journal of Medical Imaging. Vol. 8, No. 1, 2019, pp. 1-5. doi: 10.11648/j.ijmi.20200801.11

Received: December 13, 2019; Accepted: December 30, 2019; Published: January 7, 2019

\begin{abstract}
Purpose: To determine the type and frequency of characteristic MRI changes of the plantaris fascia in patients with painful heel and wear high heel shoes. Materials and Methods: 40 patients with painful heel, wearing high heels, their age raged from 25-50 years, underwent MR imaging. A control group included 20 subjects with no history of painful heel \& not using high heels were included. Associations between the presence of palnter fasciitis, high heel, and body mass index, height of the heel, MRI imaging, self-reported co-morbidities and current heel pain were then explored. MR images were obtained with a 1.5-T superconducting MR imager with a 5-inch (13-cm) standard small flexible surface coil. Results: Thirty cases (75\%) of the clinically suspected of plantar fasciitis was established by MR imaging. The most common finding was a peritendinous edema at the calcaneal insertion site which was found in all 30 patients. In 16 patients (53\%), an intratendinous signal intensity increase of the plantar fascia could be observed. Compared to the control group (mean thickness $3.3 \mathrm{~mm}$ ) the plantar fascia showed significant thickening in the thirty MR positive patients (mean thickness $7.7 \mathrm{~mm}$ ). Conclusion: Planter fasciitis is common in high heel using women. Besides thickening of the plantar fascia, intratendinous signal intensity increase and peritendinous edema close to the plantar fascia are characteristic signs of plantar fasciitis on MRI. Both signs can reliably be seen on STIR sequences only.
\end{abstract}

Keywords: MRI, Plantaris Fascia, High Heel Shoes, Painful Heel

\section{Introduction}

Women are interested to wear high-heeled shoes to increase their attractiveness. High-heeled shoes might create harmful effects to the musculoskeletal system. [1] Furthermore earlier studies proved that the function of foot and lower extremity will be changed due to wearing high heeled shoes. [2] Plantar fasciitis (also known as plantar fasciopathy or jogger's heel) is a common painful disorder affecting the heel and underside of the foot. [3] It is a disorder of the insertion site of ligament on the calcaneous bone and is characterized by scarring, inflammation, or structural breakdown of the foot's plantar fascia. It is often caused by overuse injury of the plantar fascia. [4]

Plantar fasciitis is the most common injury of the plantar fascia and is the most common cause of heel pain. It is commonly associated with excessive inward rolling of the foot as in high heel. Individuals with plantar fasciitis often have difficulty with dorsiflexion of the foot. Though plantar fasciitis was originally thought to be an inflammatory process, newer studies have demonstrated structural changes more consistent with a degenerative process or repetitive minor trauma. As a result of this new observation, many in the academic community have stated that the condition should be renamed plantar fasciosis. [5]

Understanding of the normal anatomy of the plantar aponeurosis (PA) and familiarity with pathologic conditions are required for an accurate evaluation of the patient with heel pain. [6]

The output of this research is describing the effect of wearing high heel on plantar fascia. In this study, we evaluated the diagnostic capabilities of magnetic resonance (MR) imaging in the assessment of the PA in correlation with the use of high heel.

\section{Material \& Method}

MRI study was conducted on 40 patients with a mean age of $40 \pm 7.2$ years. Twenty control volunteers, age-matched and healthy also were underwent the same protocol of MRI study. All our 40 patients were using high heels as the following criteria: length of the heel not less that 2.5 inches, a duration 
mean of 4 hours /day, 5 days/ week, with average body mass index $25-30 \%$. The control group were not wearing high heel $\&$ had no painful heel.

Patients with the following criteria were excluded from our study: over weight, abnormal shape of the foot as high arched foot, and sportive runners. All the examined patients were laboratory investigated for blood glucose level, rheumatoid factor, uric acid \& ESR. Also plain x-ray foot was done in different direction for exclusion of other causes of painful heel. Four patients were suspected to have tarsal tunnel syndrome \& nerve conduction study was done to exclude this cause.

Clinical orthopedic examination revealed heel pain with the following criteria: the pain starts with initial weight bearing after a period of time \& increases upon rising in morning, on palpation, tenderness was at the inferior aspect of the heel,

MR images were obtained with a $1.5-\mathrm{T}$ superconducting MRI (Signa; GE Medical Systems, Milwaukee, Wis) and ( Optima MR 360, Toshiba Medical Systems) at MRI units, with a 5-inch $(13-\mathrm{cm})$ standard small flexible surface coil.

Routine ankle MR imaging was performed in the axial, coronal, and sagittal planes. The foot is imaged in the oblique coronal plane, oblique axial plane, and oblique sagittal plane. T1-weighted (repetition time msec/echo time $\mathrm{msec}=600 / 20$ ) and T2-weighted $(2,000 / 20,80)$ Fat suppression techniques, fat-suppressed proton-density-weighted imaging \& shortinversion-time inversion recovery (STIR) sequences (3611/48; inversion time $\mathrm{msec}=100-150)$.

The patients were in supine position with the foot in about $20^{\circ}$ of plantar flexion; this is specially helpful for the following reasons: it accentuates the fat plane between the tendons specially the peroneal tendons $\&$ decreases the effect of the magic angle.

The imaging data were reviewed by two radiologists (with more than five years of experience) blinded of the patients clinical pictures; and then they nearly reached a consensus opinion. The regional ethics committee approved the study and written informed consent were obtained from all participants.

\section{Results}

The MR imaging features of our 40 clinical cases are listed in the Table 1. Ten cases were excluded due to presence of other bony lesions as enthesopathy, spurs \& tarsal tunnel syndrome, also of the 20 control cases one case showed mild edema denoting mild form of plantar fascitis.

MR imaging criteria in 3 patients, one patient aged 30 years, the other two at age group (46-55) aging $47 \& 50$ years respectively, these patients were having partial tear of the PS, showed partial high T2-weighted signal intensity of the plantar fascia. In the three patients, abnormal thickening of the PA at the site of partial disruption was evident. Tears involved the proximal PA was present in one case and no tear at the middle PA was observed. The three cases showed edema in perifascial soft tissue with high signal intensity on T2 WI. Fascial thickening \& scar tissue was depicted with low signal intensity on T2 WI, and STIR images.

MR imaging studies in the 27 patients with plantar fasciitis, 10 patients at age group (25-35), 11 at age group (36-45) \& 6 at age group (46-55) revealed the signal intensity changes of perifascial edema either superficial to $(n=11)$ or both superficial and deep to $(n=16)$ the PA. In 13 patients, the PA exhibited abnormal intrafascial high signal intensity on T2weighted images, STIR images, or both. The PA was abnormally thickened $(5-7 \mathrm{~mm})$ in 20 patients. (Table 1 )

Table 1. MRI findings in patients and control group.

\begin{tabular}{lll}
\hline MRI findings & Pt. (no. 40) & Control (no. 20) \\
\hline Rupture of PA & 3 & \\
Partial disruption & 3 & \\
Soft tissue edema & 3 & \\
Facial thickening & 3 & \\
Plantar faciaitis & 27 & 1 \\
Intra fascial edema & 13 & \\
Perifascial edema & 27 & 1 \\
Fascial thickening & 20 & \\
& 30 & \\
\hline
\end{tabular}

20 healthy non complaining control group were examined, one person showed superficial edema of the plantar fascia denoting mild fasciitis, while the rest of the control group showed no abnormality.

Accordingly, out of the 40 patients; 10 cases (25\%) were clinically complaining but radiologically free. On the other hand, one of the control non complaining group turned to have mild plantar fasciitis that accounts for $1 / 20=0.05 \%$.

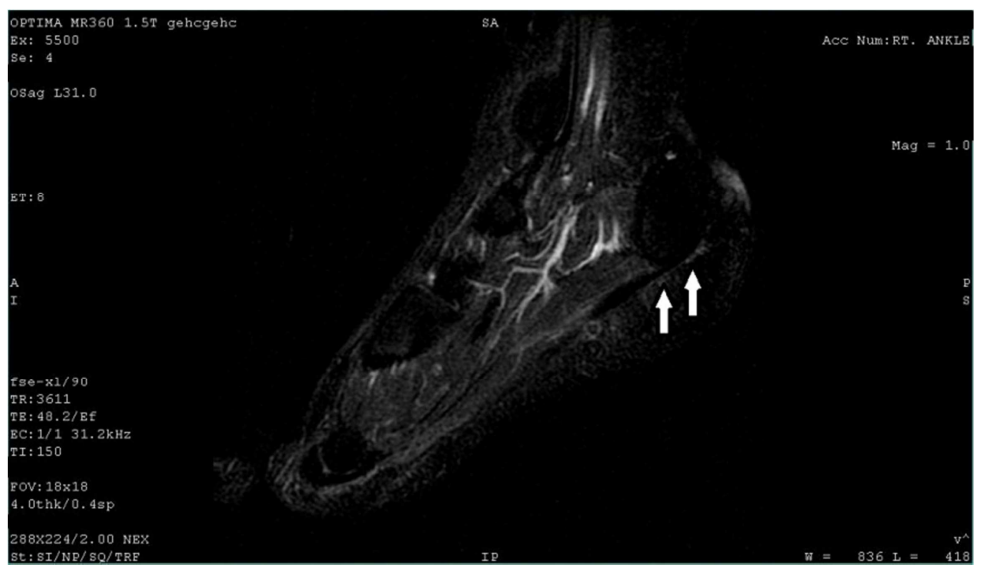

Figure 1. MRI right ankle joint, Sagittal STIR, shows abnormal peri-plantaris high signals of edema. 


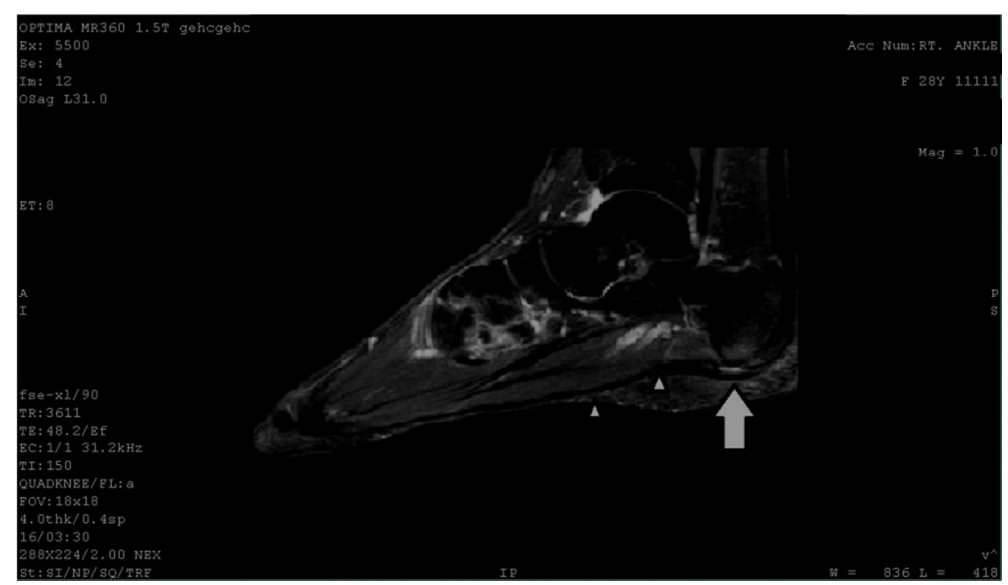

Figure 2. MRI Right ankle, Sagittal STIR, shows abnormal high signals of peri-plantaris edema deep to it (arrow) with mildly thickened plantaris facia (arrow heads).

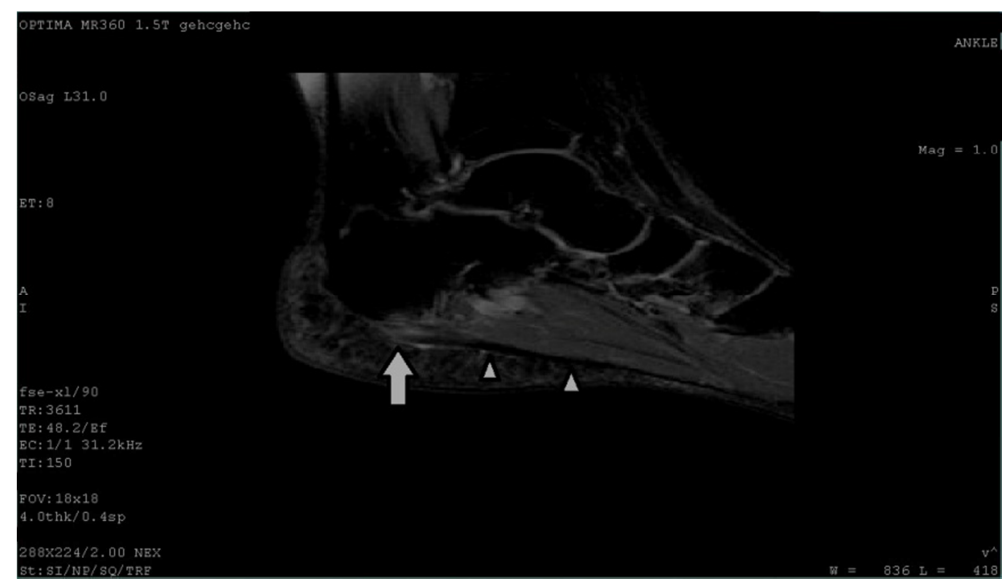

Figure 3. MRI Left ankle, Sagittal STIR, shows abnormal high signals of peri-plantaris edema superficial and deep to it as well as abnormal high signals disrupting it partially (arrow) with mildly thickened plantaris facia (arrow head).

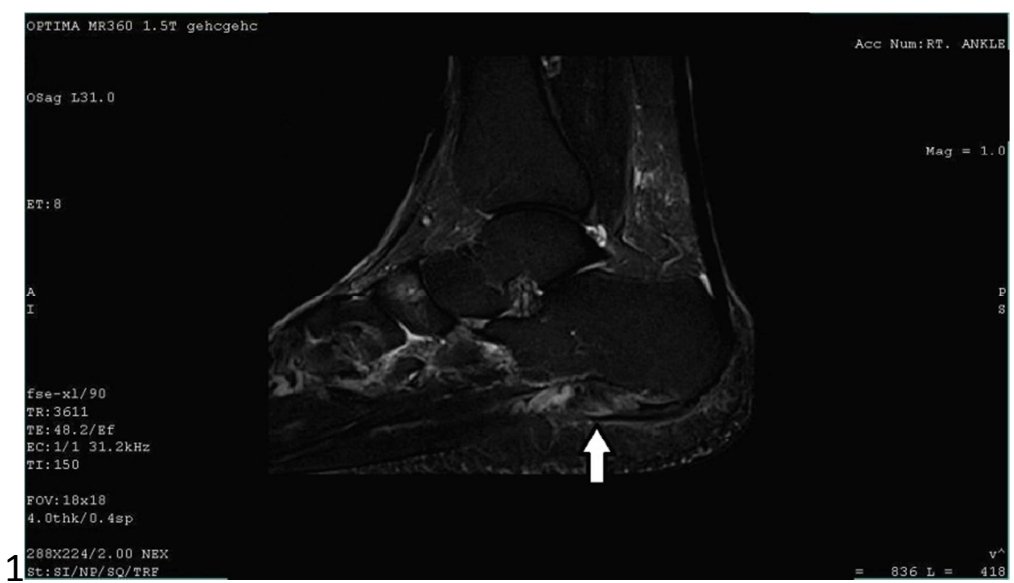

Figure 4. MRI Right ankle, Sagittal STIR, shows abnormal high signals of peri-plantaris edema superficial and deep to it as well as abnormal high signals disrupting it partially (arrow).

\section{Discussion}

The plantar fascia is a thick, ligamentous connective tissue that runs from the calcaneus to the ball of the foot. This tight tissue helps to maintain the arch of the foot and transmits weight across the foot as person walks or runs. That's why tremendous stress is placed on the plantar fascia. [7]
Heel pain is a very common complaint in adults especially in females. It is estimated that more than one million patients seek treatment annually for this condition. The plantar fasciitis is thought to be the most common cause \& is mainly due to biomechanical overuse, thus creating micro-tears at the calcaneal enthesis. [8]

Some experts have deemed this condition "plantar 
fasciosis" implying that its etiology is a more chronic degenerative process versus acute inflammation. [9] Far and away the most common cause of plantar fascia pain is faulty biomechanics of the foot or leg. Faulty biomechanics causes the foot to sustain increased or prolonged stresses over and above those of routine ground contacts. [10]

High-heeled shoes can limit proprioception where the heel hits the ground first followed by the toe. It has been shown that high heeled stiff shoes restrict the inversion/eversion of the foot, which can lead to an increase in rearfoot prontion, therefore showing that the stiffer the shoe, the more the natural foot motion is restricted. [11]

Not only is the normal foot motion restricted by shoes, it has also been shown that wearing high heeled shoes restricts other biomechanics aspects of the foot. [11] Forefoot to rearfoot eversion/inversion and abduction/adduction were restricted by shoes, as well as forefoot spreading, which is important for comfort as well as natural gait, and forefoot pronation during push-off. [12]

Plantar fasciosis can be confused with a condition called tarsal tunnel syndrome. In tarsal tunnel syndrome, the tibial nerve is trapped and pinched as it passes through the tarsal tunnel. This may cause symptoms similar to the pain of a plantar fasciosis/fasciitis, in this study, the suspected cases to have tarsal tunnel syndrome were investigated with nerve conduction study \& were all free. [13]

In all our patients suspected of having plantar fasciitis, high signal intensity consistent with edema in the perifascial soft tissue was appreciated on T2-weighted images \& STIR images as well. Our findings are in agreement with those of previous studies, in which the perifascial edema was considered the most common manifestation of plantar fasciitis. [14] In our patients with plantar fasciitis, soft-tissue edema superficial and deep to the PA was the dominant abnormal imaging finding in $90 \%$ of cases, that frequency for edema superficial to the PA was reported previously.

The second most common MR imaging finding of plantar fasciitis, seen in $66 \%$ of our patient, was mild thickening of the PA, corresponding to the presence of granulation tissue. This finding was more common in our study than was previously reported. [14]

Then, the following MR imaging finding of plantar fasciitis, observed in $23 \%$ of our cases, was increased signal intensity within the involved plantar fascia on T2-weighted and STIR images; this finding was consistent with edema, this findings did not match with the other studies, we believe that the difference may be contributed to the different number of patient $\&$ the plenty of exclusion criteria. [14]

In patients with a clinical diagnosis of partial rupture of the PA, MR imaging allowed diagnosis of the case, exact localization, and determination of severity of the lesion with regard to the thickness of the affected PA.

In the examined volunteers with age matching not wearing high heels, one case for mild edema \& plantar fasciitis was seen only among the group, raising the consideration to the effect of high heel on the PA.

As we preceded, the causes of plantaris fascia pain were lots \& we tried to exclude some causes both by clinical examination \& laboratory investigations, so that we come to a conclusion that a relation is present between the presence of plantar fascial pathology \& high heel in absence of other obvious causes of heel pain.

\section{Conclusion}

In conclusion, MRI was helpful in diagnosis of pathology of the plantar fascia in our patients with a clinical diagnosis of rupture of the PA, MR imaging allowed demonstration, exact localization, and determination of severity of the lesion with regard to the thickness of the affected PA. Depicting the relation between the complain, physical examination \& the presence of high heel in absence of any other cause for the presence of either PA rupture or fasciitis/fasciosis, \& drawing a line correlating the high heel \& plantar fascial pathology.

We recognize that although the patients show the similar symptoms \& similar imaging findings, yet the implication of this examination over a larger population may help support our conclusion.

\section{References}

[1] A. Ahmady, E. Soodmand, I. Soodmand, T. L Milani: The effect of various heights of high-heeled shoes on foot arch deformation: Finite element analysis. Journal of Foot and Ankle Research. 2014; 7 (Suppl 1): A78.

[2] Yu J.: Biomechanical simulation of high-heeled shoe donning and walking. Journal of Biomechanics. 2013, 46 (12): 2067-2074.

[3] Sullivan J, Pappas E, Burns J.: Role of mechanical factors in the clinical presentation of plantar heel pain: Implications for management. Foot (Edinb). 2019 Sep 3; 42.

[4] J. D. Goff and R. Crawford: Diagnosis and Treatment of Plantar Fasciitis. American Family Physician. 2012, 84 (6).

[5] Thomas JL, Christensen JC, and Kravitz SR.: The diagnosis and treatment of heel pain: a clinical practice guidelinerevision. J Foot Ankle Surg. 2010; 49 (3 suppl): S1-S19.

[6] Eugene G. McNally, and Shilpa Shetty.: Plantar Fascia: Imaging Diagnosis and Guided Treatment. Semin Musculoskelet Radiol. 2010; 14: 334-343.

[7] Hedrick MR.: The plantar aponeurosis. Foot Ankle. 1996; 17: 646-649.

[8] Riddle DL, Schappert SM. Volume of ambulatory care visits and patterns of care for patients diagnosed with plantar fasciitis: a national study of medical doctors. Foot Ankle Int. 2004; 25 (5): 303-310.

[9] Karr SD.: Subcalcaneal heel pain. Orthop Clin North Am. 1994; 25: 161-173.

[10] Lemont H, Ammirati KM, and Usen N.: Plantar fasciitis: a degenerative process (fasciosis) without inflammation. J Am Podiatr Med Assoc. 2003; 93 (3): 234-237.

[11] Yu J, et al: Development of a finite element model of female foot for high-heeled shoe design. Clinical Biomechanics. 2008, 23: S31-S38. 
[12] Esenyel M, et al: Kinetics of high-heeled gait. Journal of the American Pediatric Medical Association. 2003, 93 (1): 27-32.

[13] Bailie DS, Kelikian AS.: Tarsal tunnel syndrome: diagnosis, surgical technique, and functional outcome. Foot Ankle. 1998; 19: 65-72.
[14] Grasel R, Schweitzer M, Kovalovich A, et al.: MR imaging of plantar fasciitis: edema, tears, and occult marrow abnormalities correlated with outcome. AJR. 1999; 173: 699701. 